\title{
On the Location of Roots of Independence Polynomials
}

J.I. BROWN*

brown@mscs.dal.ca Department of Mathematics and Statistics and Faculty of Computer Science, Dalhousie University, Halifax, Nova Scotia, Canada B3H $3 J 5$

C.A. HICKMAN ${ }^{\dagger}$

chickman@fields.utoronto.ca

The Fields Institute for Research in Mathematical Sciences, Toronto, Ontario, Canada M5T 3JI

R.J. NOWAKOWSKI ${ }^{\ddagger}$

rjn@mscs.dal.ca

Department of Mathematics and Statistics, Dalhousie University, Halifax, Nova Scotia, Canada B3H 3J5

Received August 20, 2001; Revised March 19, 2003

Abstract. The independence polynomial of a graph $G$ is the function $i(G, x)=\sum_{k>0} i_{k} x^{k}$, where $i_{k}$ is the number of independent sets of vertices in $G$ of cardinality $k$. We prove that real roots of independence polynomials are dense in $(-\infty, 0]$, while complex roots are dense in $\mathbb{C}$, even when restricting to well covered or comparability graphs. Throughout, we exploit the fact that independence polynomials are essentially closed under graph composition.

Keywords: graph, independence, polynomial, roots

\section{Introduction}

For a graph $G$ with independence number $\beta$, let $i_{k}$ denote the number of independent sets of vertices of cardinality $k$ in $G(k=0,1, \ldots, \beta)$. Several papers exist (cf. [2, 6, 9, 11, 20]) on the independence sequence $\left(i_{1}, i_{2}, \ldots i_{\beta}\right)$ of a graph (or its complement), exploring various such problems. The independence polynomial of $G$,

$$
i(G, x)=\sum_{k=0}^{\beta} i_{k} x^{k}
$$

is the generating polynomial for the sequence. The path $P_{4}$ on 4 vertices, for example, has one independent set of cardinality 0 (the empty set), four independent sets of cardinality 1 ,

*Partially supported by a grant from NSERC.

${ }^{\dagger}$ Partially supported by an NSERC postdoctoral fellowship and a research grant from the University College of Cape Breton.

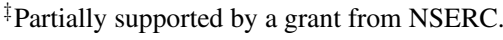


and three independent sets of cardinality 2 ; its independence polynomial is then $i\left(P_{4}, x\right)=$ $1+4 x+3 x^{2}$.

As is the case with other graph polynomials, such as chromatic polynomials (cf. $[7,28]$ ), matching polynomials $[12,13]$, and others, it is natural to consider the nature and location of the roots. Interesting in their own right, they can shed some light on the underlying combinatorics as well. One line of research in the roots of chromatic polynomials has been determining the topological closures of both the real and complex roots of the set of all chromatic polynomials. It was shown between the works of Jackson [24] and Thomassen [31] that the closure of the set of real roots of chromatic polynomials is $\{0\} \cup\{1\} \cup[32 / 27, \infty)$. Until very recently, it was not known if the closure of the set of chromatic roots in the complex plane has positive measure. Sokal [29] has shown that, in fact, chromatic roots are dense in the entire complex plane. In this paper, we shall (for the first time) answer these same questions for the roots of independence polynomials. Further results on independence sequences and polynomials can be found in [6, 10, 14-19].

We shall exploit the following result, a more general version of which was proved for dependence polynomials in [10]. For two graphs $G$ and $H$, let $G[H]$ be the graph with vertex set $V(G) \times V(H)$ and such that vertex $(a, x)$ is adjacent to vertex $(b, y)$ if and only if $a$ is adjacent to $b$ (in $G$ ) or $a=b$ and $x$ is adjacent to $y$ (in $H$ ). The graph $G[H]$ is the lexicographic product (or composition) of $G$ and $H$, and can be thought of as the graph arising from $G$ and $H$ by substituting a copy of $H$ for every vertex of $G$.

Theorem 1 Let $G$ and $H$ be graphs. Then the independence polynomial of $G[H]$ is

$$
i(G[H], x)=i(G, i(H, x)-1) .
$$

Proof: By definition, the polynomial $i(G, i(H, x)-1)$ is given by

$$
\sum_{k=0}^{\beta_{G}} i_{k}^{G}\left(\sum_{j=1}^{\beta_{H}} i_{j}^{H} x^{j}\right)^{k}
$$

where $i_{k}^{G}$ is the number of independent sets of cardinality $k$ in $G$ (similarly for $i_{k}^{H}$ ).

Now, an independent set in $G[H]$ of cardinality $l$ arises by choosing an independent set in $G$ of cardinality $k$, for some $k \in\{0,1, \ldots l\}$, and then, within each associated copy of $H$ in $G[H]$, choosing a nonempty independent set in $H$, in such a way that the total number of vertices chosen is $l$. But the number of ways of actually doing this is exactly the coefficient of $x^{l}$ in (2), which completes the proof.

By applying (1) to the right families of graphs, we will be able to determine the closures of real and complex 'independence roots'. Specifically, real independence roots are dense on the negative real axis, while complex independence roots are dense in the entire complex plane, even for such restricted families as well covered graphs and comparability graphs. This is in contrast to independence polynomials of line graphs, which are just matching generating polynomials (cf. [25]) and thus have only negative real roots. 
We shall have occasion to make use of an easy recursive formula for calculating independence polynomials.

Proposition $1[6,23]$ For any vertex $v$ of a graph $G$,

$$
i(G, x)=i(G-v, x)+x \cdot i(G-[v], x) .
$$

where [ $v]$, the closed neighbourhood of $v$, consists of $v$, together with all vertices incident with $v$.

Proof: For $k \geq 1$, an independent set of $k$ vertices in $G$ either contains $v$ or does not. There are $i_{k-1}^{G-[v]}$ that do, and $i_{k}^{G-v}$ that do not. Thus, for each $k \geq 1$, the coefficient of $x^{k}$ is the same in both sides of the above eqnarray; and both sides clearly have constant term 1. The two polynomials are therefore equal.

\section{Background: Recursive families of polynomials}

Before we proceed onto a discussion of the roots of independence polynomials, we need to state (in detail) an analytic result on particular families of polynomials (namely, recursive familes). We begin with the following definition.

Definition 1 If $\left\{f_{n}(x)\right\}$ is a family of (complex) polynomials, we say that a number $z \in \mathbb{C}$ is a limit of roots of $\left\{f_{n}(x)\right\}$ if either $f_{n}(z)=0$ for all sufficiently large $n$ or $z$ is a limit point of the set $\mathcal{R}\left(\left\{f_{n}(x)\right\}\right)$, where $\mathcal{R}\left(\left\{f_{n}(x)\right\}\right)$ is the union of the roots of the $f_{n}(x)$.

Now (as in [3]) a family $\left\{f_{n}(x)\right\}$ of polynomials is a recursive family of polynomials if the $f_{n}(x)$ satisfy a homogenous linear recurrence

$$
f_{n}(x)=\sum_{i=1}^{k} a_{i}(x) f_{n-i}(x),
$$

where the $a_{i}(x)$ are fixed polynomials, with $a_{k}(x) \not \equiv 0$. The number $k$ is the order of the recurrence.

We can form from (3) its associated characteristic equation

$$
\lambda^{k}-a_{1}(x) \lambda^{k-1}-a_{2}(x) \lambda^{k-2}-\cdots-a_{k}(x)=0,
$$

whose roots $\lambda=\lambda(x)$ are algebraic functions, and there are exactly $k$ of them counting multiplicity (c.f. $[1,22])$.

If these roots, say $\lambda_{1}(x), \lambda_{2}(x), \ldots, \lambda_{k}(x)$, are distinct, then the general solution to (3) is known [3] to be

$$
f_{n}(x)=\sum_{i=1}^{k} \alpha_{i}(x) \lambda_{i}(x)^{n},
$$


with the 'usual' variant (cf. [3]) if some of the $\lambda_{i}(x)$ were repeated. The functions $\alpha_{1}(x)$, $\alpha_{2}(x), \ldots, \alpha_{k}(x)$ are determined from the initial conditions, that is, the $k$ linear equations in the $\alpha_{i}(x)$ obtained by letting $n=0,1, \ldots k-1$ in (5) or its variant. The details are found in [3].

Beraha et al. [3] proved the result below on recursive families of polynomials and their roots.

Theorem 2 [3] If $\left\{f_{n}(x)\right\}$ is a recursive family of polynomials, then a complex number $z$ is a limit of roots of $\left\{f_{n}(x)\right\}$ if and only if there is a sequence $\left\{z_{n}\right\}$ in $\mathbb{C}$ such that $f_{n}\left(z_{n}\right)=0$ for all $n$ and $z_{n} \rightarrow z$ as $n \rightarrow \infty$.

The main result of their paper characterizes precisely the limits of roots of a recursive family of polynomials.

Theorem 3 [3] Under the non-degeneracy requirements that in (5) no $\alpha_{i}(x)$ is identically zero and that for no pair $i \neq j$ is $\lambda_{i}(x) \equiv \omega \lambda_{j}(x)$ for some $\omega \in \mathbb{C}$ of unit modulus, then $z \in \mathbb{C}$ is a limit of roots of $\left\{f_{n}(x)\right\}$ if and only if either

(i) two or more of the $\lambda_{i}(z)$ are of equal modulus, and strictly greater (in modulus) than the others; or

(ii) for some $j, \lambda_{j}(z)$ has modulus strictly greater than all the other $\lambda_{i}(z)$ have, and $\alpha_{j}(z)=0$.

This result has found application to the chromatic roots of recursive families of graphs (cf. [5]), that is, families of graphs whose Tutte (and therefore chromatic) polynomials satisfy a homogeneous linear recurrence; see $[4,27]$ for some examples.

\section{Location of independence roots of some families of graphs}

As advertised, we shall now find the topological closures of real and complex independence roots. As the coefficients of any independence polynomial are positive all the way down to the constant term, it is clear that no real independence root is nonnegative. Nevertheless, we have:

Theorem 4 Complex roots of independence polynomials are dense in all of $\mathbb{C}$, while real independence roots are dense in $(-\infty, 0]$.

We will prove Theorem 4 by considering very specific families of graphs, taking lexicographic products, and examining the roots of the independence polynomials that arise. The upshot will be the truth of Theorem 4 even for some very restricted families of graphs, namely well covered and comparability graphs.

In fact, the first half of Theorem 4 follows from the second, by composing with empty graphs. Since each subset of vertices in $\bar{K}_{m}$ is independent, it follows from the Binomial Theorem that $i\left(\bar{K}_{m}, x\right)=(1+x)^{m}$. 
Theorem 5 If $\left\{G_{n}\right\}$ is a family of graphs whose real independence roots are dense in the interval $(-\infty, 0]$, then the family $\left\{G_{n}\left[\bar{K}_{m}\right]\right\}$ has independence roots that are dense in $\mathbb{C}$.

Proof: Denote by $R$ the set of real independence roots of the family $\left\{G_{n}\right\}$; by supposition, $\bar{R}=(-\infty, 0]$. Let $z \in \mathbb{C}$ and $\varepsilon>0$. We will show that there exist positive integers $n, m$ such that $i\left(G_{n}\left[\bar{K}_{m}\right], \tilde{z}\right)=0$ for some $\tilde{z}$ within an $\varepsilon$-radius of $z$. From Proposition 1, we have

$$
i\left(G_{n}\left[\bar{K}_{m}\right], x\right)=i\left(G_{n}, i\left(\bar{K}_{m}, x\right)-1\right)=i\left(G_{n},(1+x)^{m}-1\right)
$$

We may assume $z \neq-1$; thus $|z+1|>0$. Choose $m$ large enough that some $m$-th root of $-|z+1|^{m}$, say $w=|z+1| e^{\frac{i(2 k+1) \pi}{m}}$, is within an $\frac{\varepsilon}{2}$-radius of $z+1$. Choose $\delta>0$ such that $\delta<\frac{\varepsilon}{2}$ and $r=-(|z+1|+\delta)^{m}-1 \in R$ (such a $\delta$ exists, since $R$ is dense in $(-\infty, 0]$ and $-(|z+1|+\delta)^{m}-1$ is a continuous function of $\left.\delta\right)$. Then the corresponding $m$-th root of $r+1=-(|z+1|+\delta)^{m}$, namely $\tilde{w}=(|z+1|+\delta) e^{\frac{i(2 k+1) \pi}{m}}$, is within an $\varepsilon$-radius of $z+1$, as

$$
\begin{aligned}
|\tilde{w}-(z+1)| & =|(\tilde{w}-w)+(w-(z+1))| \\
& \leq|\tilde{w}-w|+|w-(z+1)| \\
& <\delta+\frac{\varepsilon}{2}<\frac{\varepsilon}{2}+\frac{\varepsilon}{2}=\varepsilon .
\end{aligned}
$$

Finally, since $r \in R$, there is a positive integer $n$ for which $i\left(G_{n}, r\right)=0$. Set $\tilde{z}=\tilde{w}-1$. Then

$$
|\tilde{z}-z|=|(\tilde{w}-1)-z|=|\tilde{w}-(z+1)|<\varepsilon,
$$

and

$$
\begin{aligned}
i\left(G_{n}\left[\bar{K}_{m}\right], \tilde{z}\right) & =i\left(G_{n},(1+\tilde{z})^{n}-1\right) \\
& =i\left(G_{n}, \tilde{w}^{m}-1\right) \\
& =i\left(G_{n},(r+1)-1\right) \\
& =i\left(G_{n}, r\right) \\
& =0
\end{aligned}
$$

completing the proof.

\subsection{Well covered graphs}

A graph is well covered if every maximal set of independent vertices has the same cardinality. The graph $C_{4}$, for instance, is well covered with independence number 2 , while $C_{6}$, a graph with independence number 3 , is not well covered, since it contains maximal independent subsets of cardinality 2 . Well covered graphs have attracted considerable attention; see [26] for an extensive survey. We omit the proof of the following simple result. 
Proposition 2 If $G$ and $H$ are well covered, then $G[H]$ is also well covered.

Denote by $[1, \beta]$ the set $\{1,2, \ldots, \beta\}$. As in [6], $L_{\beta}^{k}$ (where $k$ is a positive integer) is the 'lattice graph' with vertex set $[1, \beta]^{k}$, in which two $k$-tuples are joined by an edge if and only if they agree in a coordinate. The next result is sufficiently simple that we can state it without proof.

Proposition 3 For $\beta, k \geq 2$, the graph $L_{\beta}^{k}$ is well covered with independence number $\beta$.

The graphs $L_{\beta}^{k}$ were considered in [6], where the following was shown.

Theorem 6 [6] The independence polynomials $i\left(L_{\beta}^{k}, x\right)$ have all real, negative roots. Further, if $2^{k-1} \geq \beta \geq 1$ then the smallest root $y_{\beta}^{(k)}$ of $i\left(L_{\beta}^{k}, x\right)$ lies in the interval

$$
-\beta<y_{\beta}^{(k)}<-\beta\left(1-2^{-k}\right)
$$

By taking the lexicographic product of the $L_{\beta}^{k}$ with complete graphs, we find below that the independence roots which arise are real and dense in $(-\infty, 0]$. Complete graphs are obviously well covered (with independence number 1 ), and $i\left(K_{n}, x\right)=1+n x$. Proposition 2 then implies that $L_{\beta}^{k}\left[K_{n}\right]$ is well covered, and, by Eq. (1), $i\left(L_{\beta}^{k}\left[K_{n}\right], x\right)=i\left(L_{\beta}^{k}, n x\right)$.

Theorem 7 The independence roots of the family $\left\{L_{\beta}^{k}\left[K_{n}\right]\right\}$ are real and dense in $(-\infty, 0]$.

Proof: Since $i\left(L_{\beta}^{k}, x\right)$ has all real roots, so too does $i\left(L_{\beta}^{k}\left[K_{n}\right], x\right)=i\left(L_{\beta}^{k}, n x\right)$. Let $s \in$ $(-\infty, 0]$ and $\varepsilon>0$ be given. We will show that there are positive integers $\beta, k$ and $n$ such that $i\left(L_{\beta}^{k}\left[K_{n}\right], x\right)=i\left(L_{\beta}^{k}, n x\right)$ has a root in the interval $(s-\varepsilon, s+\varepsilon)$. Begin by choosing a positive integer $n$ large enough that the interval $n \cdot(s-\varepsilon, s+\varepsilon) \equiv(n s-n \varepsilon, n s+n \varepsilon)$ contains some integer $\beta \leq-2$. By Theorem 6 , there is a number $k$ such that $i\left(L_{\beta}^{k}, x\right)$ has a root $r$ in that interval. Then $r / n \in(s-\varepsilon, s+\varepsilon)$, and

$$
i\left(L_{\beta}^{k}\left[K_{n}\right], \frac{r}{n}\right)=i\left(L_{\beta}^{k}, n \cdot \frac{r}{n}\right)=i\left(L_{\beta}^{k}, r\right)=0
$$

completing the proof.

The following is a direct consequence of Theorems 5 and 7.

Corollary 1 The independence roots of the family $L_{\beta}^{k}\left[K_{n}\right]\left[\bar{K}_{m}\right]$ are dense in $\mathbb{C}$.

The family $L_{\beta}^{k}\left[K_{n}\right]\left[\bar{K}_{m}\right]$ is well covered, since empty graphs $\bar{K}_{m}$ are obviously well covered. Thus, Theorem 7 and Corollary 1 imply that Theorem 4 is true even when restricting to well covered graphs. 


\subsection{Comparability graphs}

A simple graph $G$ is a comparability graph if it has a transitive orientation, that is, an orientation of its edges such that if $x \rightarrow y$ and $y \rightarrow z$ then $x \rightarrow z$. Comparability graphs are also closed under graph composition.

Proposition 4 If $G$ and $H$ are comparability graphs, then $G[H]$ is also a comparability graph.

Proof: Orient the graph $G[H]$ by $(a, x)<(b, y)$ if and only if $a<b$ (in $G)$ or $a=b$ and $x<y$ (in $H$ ). This is a transitive orientation of $G[H]$. For suppose $(a, x)<(b, y)$ and $(b, y)<(c, z)$. If $a=b=c$, then $x<y$ and $y<z$, and so $x<z$ by transitivity of $H$, whence $(a, x)<(c, z)$. If instead $a=b<c$ or $a<b=c$ or $a<b<c$, then $a<c$ by transitivity of $G$, and so $(a, x)<(c, z)$. This completes the proof, as there are no other possibilities.

Contained in the collection of comparability graphs are paths, complete graphs and empty graphs. We omit the proof of this basic fact.

Proposition 5 Paths, complete graphs and empty graphs are all comparability graphs.

Together with Proposition 4, this implies:

Corollary 2 The graphs $P_{n_{1}}\left[K_{n_{2}}\right]$ and $P_{n_{1}}\left[K_{n_{2}}\right]\left[\bar{K}_{n_{3}}\right]$ are comparability graphs.

Analogous to what we did for well covered graphs, we will show that the family $\left\{P_{n_{1}}\left[K_{n_{2}}\right]\right\}$ has real independence roots which are dense in $(-\infty, 0]$. It then follows from Theorem 5 that the complex independence roots of the family $\left\{P_{n_{1}}\left[K_{n_{2}}\right]\left[\bar{K}_{n_{3}}\right]\right\}$ are dense in all of $\mathbb{C}$.

We begin with paths, themselves.

Theorem 8 The independence roots of the family $\left\{P_{n}\right\}$ are real and dense in $\left(-\infty,-\frac{1}{4}\right]$.

Proof: Since $P_{n}$ is the line graph of $P_{n+1}, M\left(P_{n+1}, x\right)=x^{n} i\left(P_{n},-1 / x^{2}\right)$, the former being the matching polynomial of $P_{n+1}$, and matching polynomials are known [21] to have only real roots. It follows that $i\left(P_{n}, x\right)$ has only real roots as well. The reduction in Proposition 1 for calculating independence polynomials gives

$$
i\left(P_{n}, x\right)=i\left(P_{n-1}, x\right)+x \cdot i\left(P_{n-2}, x\right) \quad(l \geq 3)
$$

and so the family $\left\{i\left(P_{n}, x\right)\right\}$ is recursive; the initial conditions are $i\left(P_{1}, x\right)=1+x$ and $i\left(P_{2}, x\right)=1+2 x$. Solving, we find

$$
i\left(P_{n}, x\right)=\alpha_{1}(x) \lambda_{1}(x)^{n}+\alpha_{2}(x) \lambda_{2}(x)^{n},
$$


where

$$
\lambda_{1}(x)=\frac{1+\sqrt{1+4 x}}{2}, \quad \lambda_{2}(x)=\frac{1-\sqrt{1+4 x}}{2}
$$

and

$$
\alpha_{1}(x)=\frac{\sqrt{1+4 x}+(1+2 x)}{2 \sqrt{1+4 x}}, \quad \alpha_{2}(x)=\frac{\sqrt{1+4 x}-(1+2 x)}{2 \sqrt{1+4 x}} .
$$

The non-degeneracy conditions of the Beraha-Kahane-Weiss theorem (Theorem 3) are therefore satisfied, and part (i) of that theorem implies that among the limits of roots are those $z$ for which

$$
\left|\lambda_{1}(z)\right|=\left|\lambda_{2}(z)\right|,
$$

which simplifies to

$$
|1+\sqrt{1+4 z}|=|1-\sqrt{1+4 z}|
$$

implying that $\sqrt{1+4 z}$ is purely imaginary. Thus $1+4 z \leq 0$, i.e., $z \leq-1 / 4$, which is what we wanted to show.

By composing with complete graphs, we can fill up the rest of the negative real axis.

Theorem 9 The independence roots of the family $P_{n_{1}}\left[K_{n_{2}}\right]$ are real and dense in $(-\infty, 0]$.

Proof: From Theorem 8, independence roots of the graphs $P_{n_{1}}\left[K_{1}\right]=P_{n_{1}}$ are real and dense in $(-\infty,-1 / 4]$. Let $s \in(-1 / 4,0)$ and $\varepsilon>0$ be given. Then there are positive integers $n_{1}$ and $n_{2}$ for which $i\left(P_{n_{1}}\left[K_{n_{2}}\right], x\right)=i\left(P_{n_{1}}, n_{2} x\right)$ has a root in $(s-\varepsilon, s+\varepsilon)$. Indeed, choose $n_{2}$ large enough that $n_{2} s \leq-1 / 4$. Then, from Theorem 8 , there is a number $n_{1}$ such that $i\left(P_{n_{1}}, x\right)$ has a root $r \in n_{2} \cdot(s-\varepsilon, s+\varepsilon) \equiv\left(n_{2} s-n_{2} \varepsilon, n_{2} s+n_{2} \varepsilon\right)$. But then $\frac{1}{n_{2}} \cdot r \in(s-\varepsilon, s+\varepsilon)$ and $i\left(P_{n_{1}}\left[K_{n_{2}}\right], \frac{1}{n_{2}} \cdot r\right)=i\left(P_{n_{1}}, n_{2} \cdot \frac{r}{n_{2}}\right)=i\left(P_{n_{1}}, r\right)=0$, completing the proof.

By Theorem 5, compositions with empty graphs will then fill up the complex plane.

Corollary 3 The independence roots of the graphs $P_{n_{1}}\left[K_{n_{2}}\right]\left[\bar{K}_{n_{3}}\right]$ are dense in $\mathbb{C}$.

Hence, Theorem 4 remains true when we restrict to comparability graphs. 


\section{Concluding remarks}

It may be of interest to study the independence roots of yet further classes of graphs. Some common ones include chordal, interval, claw-free, and line graphs.

It is known (cf. [32]) that interval graphs are chordal, and line graphs are claw-free. The reader can verify that the graphs $P_{n_{1}}\left[K_{n_{2}}\right]$ are chordal, interval, and claw-free, while graphs $P_{n_{1}}\left[K_{n_{2}}\right]\left[\bar{K}_{n_{3}}\right]\left(n_{3} \geq 2\right)$ are neither. Thus, real independence roots of chordal, interval, or claw-free graphs are dense in $(-\infty, 0]$, while further investigation would be necessary to determine where the complex roots of those families lie. Finally, since independence polynomials of line graphs are essentially just matching polynomials, their roots are real and negative [21]. Paths $P_{n}$ are line graphs, while $P_{n_{1}}\left[K_{n_{2}}\right]$ and $P_{n_{1}}\left[K_{n_{2}}\right]\left[\bar{K}_{n_{3}}\right]$ are not. Therefore, line graphs have independence roots which are dense in at least $\left(-\infty,-\frac{1}{4}\right]$, but it remains to be seen whether they are in fact dense on the entire negative real axis.

\section{Acknowledgments}

The authors would like to thank the referees for their insightful comments.

\section{References}

1. L. Ahlfors, Complex Analysis, 3rd edition, McGraw-Hill, New York, 1979.

2. Y. Alavi, P. Malde, A.J. Schwenk, and P. Erdös, "The vertex independence sequence of a graph is not constrained," Congr. Numer. 58 (1987), 15-23.

3. S. Beraha, J. Kahane, and N. Weiss, "Limits of zeros of recursively defined families of polynomials," in Studies in Foundations and Combinatorics: Advances in Math., Supplementary Studies, vol. 1, G. Rota (Ed.), Academic Press, New York, 1978.

4. S. Beraha, J. Kahane, and N.J. Weiss, "Limits of chromatic zeros of some families of graphs," J. Combin. Theory Ser. B 28 (1980), 52-65.

5. N.L. Biggs, R.M. Damerell, and D.A. Sands, "Recursive families of graphs," J. Combin. Theory Ser. B 12 (1972), 123-131.

6. J.I. Brown, K. Dilcher, and R.J. Nowakowski, "Roots of independence polynomials of well covered graphs," J. Algebraic Combin. 11 (2000), 197-210.

7. J.I. Brown and C.A. Hickman, "On chromatic roots of large subdivisions of graphs," Discrete Math. 242 (2002), 17-30.

8. L. Comtet, Advanced Combinatorics, Reidel Pub. Co., Boston, 1974.

9. D.C. Fisher, "Lower bounds on the number of triangles in a graph," J. Graph Theory 13 (1989), 505-512.

10. D.C. Fisher and A.E. Solow, "Dependence polynomials," Discrete Math. 82 (1990), 251-258.

11. D.C. Fisher and J. Ryan, "Bounds on the number of complete subgraphs," Discrete Math. 103 (1992), 313320.

12. C.D. Godsil, "Real graph polynomials," in Progress in Graph Theory, J.A. Bondy and U.S.R. Murty (Eds.), Academic Press, Toronto, 1984.

13. C.D. Godsil and I. Gutman, "On the theory of matching polynomials," J. Graph Theory, 5 (1981), $137-144$.

14. I. Gutman, "On independent vertices and edges of belt graphs," Publ. Inst. Math. (Belgrade) 59 (1996), 11-17.

15. I. Gutman, "Numbers of independent vertex and edge sets of a graph: Some analogies," Graph Theory Notes (New York) 22 (1992), 18-22.

16. I. Gutman, "Some analytic properties of the independence and matching polynomials," Match. 28 (1992), $139-150$. 
17. I. Gutman, "An idendity for the independence polynomials of trees," Publ. Inst. Math. (Belgrade) 50 (1991), 19-23.

18. I. Gutman, "On independent vertices and deges in a graph," in Topics in Combinatorics and Graph Theory, R. Bodendeik and R. Henn (Eds.), Physica-Verlag, Heidelberg, 1990.

19. I. Gutman, "Graphs with maximimum and minimum independence numbers," Publ. Inst. Math. (Belgrade) 34 (1983), 73-79.

20. Y.O. Hamidoune, "On the number of $k$-sets in a claw free graph,” J. Combin. Theory Ser. B 50 (1990), 241-244.

21. O.J. Heilmann and E.H. Lieb, "Theory of monomer-dimer systems," Commun. Math. Phys. 25 (1972), 190232.

22. E. Hille, Analytic Function Theory, Ginn \& Co., Boston, 1959.

23. C. Hoede and X. Li, "Clique polynomials and independent set polynomials of graphs," Discrete Math. 25 (1994), 219-228

24. B. Jackson, "A zero-free interval for the chromatic polynomials of graphs," Combin. Probab. Comp. 2 (1993), 325-336.

25. L. Lovasz and M.D. Plummer, Matching Theory, North-Holland Publishing Co., Amsterdam, 1986.

26. M.D. Plummer, "Well covered graphs: A survey," Quaestiones Math. 8 (1970), 91-98.

27. R.C. Read and G.F. Royle, "Chromatic roots of families of graphs," in Graph Theory, Combinatorics, and Applications, Y. Alavi et al. (Eds.), Wiley, New York, 1991.

28. R.C. Read and W.T. Tutte, "Chromatic polynomials," in Selected Topics in Graph Theory 3, Y.W. Beineke and R.J. Wilson (Eds.), Academic Press, New York, 1988.

29. A.D. Sokal, "Chromatic polynomials, Potts models and all that," Physica A 279 (2000), 324-332.

30. R.P. Stanley, "Log-concave and unimodal sequences in algebra, combinatorics, and geometry," Ann. New York Acad. Sci. 576 (1989), 500-534.

31. C. Thomassen, "The zero-free intervals for chromatic polynomials of graphs," Combin. Probab. Comp. 6 (1997), 4555-4564.

32. D.B. West, Introduction to Graph Theory, Prentice-Hall, New Jersey, 1996. 